\title{
At-risk gambling in patients with severe mental illness: Prevalence and associated features
}

\author{
ANNALISA BERGAMINI ${ }^{1}$, CESARE TURRINA ${ }^{1,2} *$, FRANCESCA BETTINI ${ }^{1}$, ANNA TOCCAGNI ${ }^{1}$, PAOLO VALSECCHI $^{1}$, \\ EMILIO SACCHETTI ${ }^{1}$ and ANTONIO VITA ${ }^{1,2}$
}

\author{
${ }^{1}$ Department of Clinical and Experimental Sciences, University of Brescia, Brescia, Italy \\ ${ }^{2}$ Department of Mental Health, ASST Spedali Civili, Brescia, Italy
}

(Received: October 16, 2017; revised manuscript received: January 8, 2018; second revised manuscript received: April 17, 2018; accepted: April 17, 2018)

\begin{abstract}
Background and aims: The primary objective of this study was to investigate the prevalence of at-risk gambling in a large, unselected sample of outpatients attending two community mental health centers, to estimate rates according to the main diagnosis, and to evaluate risk factors for gambling. Methods: All patients attending the centers were evaluated with the Canadian Problem Gambling Index and the Mini International Neuropsychiatric Interview. Diagnoses were checked with the treating psychiatrists and after a chart review of the university hospital discharge diagnoses. Results: The rate of at-risk gambling in 900 patients was 5.3\%. In those who gambled over the last year, $10.1 \%$ were at-risk gamblers. The rates in the main diagnostic groups were: $4.7 \%$ schizophrenia and related disorders, $4.9 \%$ bipolar disorder, $5.6 \%$ unipolar depression, and $6.6 \%$ cluster B personality disorder. In $52.1 \%$ of the cases, at-risk gambling preceded the onset of a major psychiatric disorder. In a linear regression analysis, a family history of gambling disorder, psychiatric comorbidities, drug abuse/dependence, and tobacco smoking were significantly associated with at-risk gambling. Discussion and conclusion: The results of this study evidenced a higher rate of at-risk gambling compared to community estimates and call for a careful screening for gambling in the general psychiatric population.
\end{abstract}

Keywords: gambling, schizophrenia, bipolar disorder, depression, personality disorders

\section{INTRODUCTION}

Research on the comorbidity of gambling problems with other psychiatric diagnoses has mainly focused on the comorbidity of gambling and substance-use problems (Bonnaire et al., 2017; Rodriguez-Monguio, Errea, \& Volberg, 2017; Wareham \& Potenza, 2010), whereas the comorbidity in patients with major psychiatric illness has been less investigated. The first published study on gambling behavior in outpatients with schizophrenia was conducted by Desai and Potenza (2009) and reported a 19\% prevalence rate. As far as any psychotic illness, only one study was published on patients with schizophrenia, bipolar illness, depressive psychosis, and schizoaffective disorder, reporting a risk of problem gambling four times higher in patients than the general population (Haydok et al., 2015).

As far as bipolar illness, a mailing survey of the Bipolar Disorder Research Network (Jones et al., 2015) on 750 participants, with a $23 \%$ completion rate, found a $10.6 \%$ overall rate of problem gambling, whereas severe risk was $2.7 \%$. Another relevant paper is the Epidemiologic Catchment Area study (McIntyre et al., 2007) that found a 13.3\% rate of problem gambling in individuals with bipolar disorder.

As for unipolar depressive patients, Kennedy et al. (2010) reported a $9.4 \%$ prevalence rate of problem gambling and in another study, Quilty, Watson, Robinson, Toneatto, and Bagby (2011) found a rate of $12.5 \%$. Some authors have also hypothesized a causal relationship in the comorbidity of depression and gambling, by investigating the time course of the two disorders (Chou \& Afifi, 2011; Dussault et al., 2016).

Screening for gambling problems in the setting of community mental health $(\mathrm{CMH})$ services may have relevant implications for a population that is generally out of reach from drug addiction services and primary care. It could also help in detecting early stages of gambling addiction and thus give room to implement primary prevention. It is reported that even subjects with low scores on screening tools for gambling do have significant dependence and social harms (Canale, Vieno, \& Griffiths, 2016). Furthermore, the high comorbidity in mood disorder patients for gambling and other addictions suggests that the detection of one condition should trigger an assessment and relevant treatment for all comorbid conditions (Kennedy et al., 2010).

\footnotetext{
* Corresponding author: Prof. Cesare Turrina; Department of Mental Health, ASST Spedali Civili 1, 25100 Brescia, Italy; Phone: +39 030 3995233; Fax: +39 030 3384089; E-mail: cesare.turrina@unibs.it
}

This is an open-access article distributed under the terms of the Creative Commons Attribution-NonCommercial 4.0 International License, which permits unrestricted use, distribution, and reproduction in any medium for non-commercial purposes, provided the original author and source are credited, a link to the CC License is provided, and changes - if any - are indicated. 
The study of the comorbidity of gambling within definite psychiatric diagnoses may shed light on the pathophysiology of comorbidities, but mixed diagnostic samples can also be informative on the burden of a complex comorbidity in the setting of psychiatric services. General psychiatric samples are of particular interest, because the well-known social, economic, and legal complications of gambling can be even more distressing in patients with a major psychiatric diagnosis and, as with other addiction disorders, may complicate the course, treatment adherence, and overall prognosis of the main psychiatric illness. To date, only few papers have been published in the general psychiatric population (Aragay et al., 2012; Zimmerman, Chelminski, \& Young, 2006).

We conducted a survey on a large psychiatric sample of patients attending two $\mathrm{CMH}$ centers in the city of Brescia, Italy, with the aim of estimating the rate of at-risk gambling in the whole sample. We also expected that rates would be different across diagnostic categories and that the associations commonly found in patients with primary gambling or in community surveys would be replicated in patients with severe mental illness.

\section{METHODS}

\section{Participants}

The study was conducted in the University Psychiatric Unit of the Department of Mental Health of the ASST Spedali Civili of Brescia, Italy. The Psychiatric Unit catchment area is about two thirds of the people living in the city of Brescia and its hinterland, totaling 250,592 inhabitants. It comprises an acute hospital ward, a rehabilitation center, medium longterm facilities, and two $\mathrm{CMH}$ centers. The psychiatrists at the $\mathrm{CMH}$ regularly visit all the psychiatric unit patients and implement comprehensive treatment plans. Patients with a primary diagnosis of substance use are cared for by separate, dedicated centers, so patients attending the $\mathrm{CMH}$ centers have a primary diagnosis of a major psychiatric disorder. The distinction of psychiatric services from services for addictions has been operating in Italy since law 833 passed in 1978. Regions have later gained major responsibility in running the health system and recently, in 2015, Regione Lombardia ruled that psychiatric and addiction services were part of the same mental health department with a single direction. In the city of Brescia, a Service for Behavioral Addictions has been operating for the past 2 years.

All patients attending the $\mathrm{CMH}$ centers from January 1 to June 30, 2016 were consecutively included in this study. Inclusion criteria were: (a) age 18-70, (b) IQ $>70$, and (c) comprehension of spoken Italian. Patients with a primary diagnosis of organic brain syndrome were excluded from the sample. All subjects were tested by psychiatrists or medical doctors with at least 4 years of clinical experience in psychiatry.

\section{Measures}

Basic sociodemographic information was collected using a standard demographic questionnaire. The clinical data collected were personal history of child-adolescent neuropsychiatric disorders, psychiatric comorbidity, alcohol abuse/dependence, substance abuse/dependence, smoking, suicide attempt in the last year and lifetime, and family history of gambling disorder.

All participants completed the 9-item Problem Gambling Severity Index of the Canadian Problem Gambling Index (CPGI; Ferris \& Wynne, 2001) and the Mini International Neuropsychiatric Interview (MINI), a brief structured interview for the major axis-I psychiatric disorders in DSM-IV and ICD 10 (Sheehan et al., 1998).

The psychometric properties of the Italian version of CPGI were tested in a large community survey of over 5,000 subjects (Colasante et al., 2013). The internal consistency was elevated (Cronbach's $\alpha=.87$ ) and the confirmatory factor analysis found a single factor with good eigenvalues (4.68). The convergent validity of CPGI was also tested in the comparison with the Lie/Bet Questionnaire showing a highly significant association. Three questions were added that addressed the first game ever played, the main game in the last year, and the preferred place for gambling.

Scores on the CPGI define no-risk gambling (0), low risk (1-2), moderate risk (3-7), and problem gambling (8+). There is evidence from recent literature that an alternative system for scoring the CPGI can yield greater classification accuracy relative to clinician ratings, with the definition of "low to moderate severity problems" (1-4) and "problem gambling" (5+) (Cowlishaw, Gale, Gregory, McCambridge, \& Kessler, 2017; Williams \& Volberg, 2014). The results are reported according to different cut-off point criteria, while the comparison of prevalence estimates between diagnostic groups and with other studies of the literature is based on the 3+ threshold (Jones et al. 2015; Kennedy et al., 2010; Quilty et al., 2011), defined as "at-risk gambling."

The interrater reliability of the Italian version of the MINI was tested in 50 psychiatric outpatients (Rossi et al., 2004) and all the kappa values were above 0.73 , indicating good agreement. The MINI was supplemented with questions investigating the temporal course of the disorders.

The final decision on a patient diagnosis according to the fourth edition of Diagnostic and Statistical Manual of Mental Disorders (text revision) (American Psychiatric Association, 2000) was made after a joint evaluation of three major sources: the MINI, the treating psychiatrist, and the hospital discharge diagnosis (if present).

\section{Statistical analysis}

The analysis of associations with CPGI total score used Student's $t$-test with a 0.05 level of significance. All variables associated with gambling in the univariate analysis were entered as independent variables in a linear regression analysis, where the dependent variable was CPGI total score. A stepwise, backward selection was used to remove the least significant variable at each step and in the final model only variables with a significant $(p<.05)$ and independent association were left.

\section{Ethics}

The study procedure was carried out in accordance with the Declaration of Helsinki. The Institutional Review Board of 
the Department of Clinical and Experimental Sciences of the University of Brescia approved the study (NP2884). All subjects were informed about the study and all provided signed informed consent.

\section{RESULTS}

Out of 917 patients who were eligible for the study, 17 $(1.9 \%)$ refused to be interviewed, so that a total of 900 patients were included in the sample. Their mean age was 48.7 years $(S D=13.7), 48.3 \%$ were men, $50.2 \%$ had only an eighth-grade education or less, $35 \%$ were married, and $38 \%$ employed. Their main diagnoses were schizophrenia or related disorders $(38.3 \%)$, unipolar depression (19.8\%), bipolar disorder $(11.4 \%)$, cluster B personality disorder $(20.3 \%)$, and other disorders $(10.2 \%)$. In $35.3 \%$ of the cases, two or more psychiatric diagnoses were applied. The main sociodemographic and clinical features of our sample are reported in Table 1. The psychotropic drugs most often prescribed were $57.6 \%$ antipsychotics, $21.3 \% \operatorname{mood}$ stabilizers, and $50.0 \%$ antidepressants.

At-risk gamblers, as defined by a CPGI score of $3+$, were 5.3\% (48 subjects; $95 \%$ CI: 3.8-6.8). According to different thresholds used by recent literature $(0,1-2,3-7,8+$ or 0 , $1-4,5+)$, the following at-risk groups were detected: no risk (0) 90.6\% (88.5-92.3), low risk (1-2) 4.1\% (3.0-5.6), moderate risk (3-7) $2.0 \%$ (1.3-3.1), problem gambling (8+) $3.3 \%(2.3-4.7)$ or low to moderate severity problems (1-4) $5.3 \%$ (4.0-7.0), and problem gambling (5+) $4.1 \%$ (3.0-5.6). Among the subjects who gambled in the last year, $10.1 \%$ (7.4-12.8) were at-risk gamblers. The rate of at-risk gambling in the main psychiatric diagnoses was: schizophrenia and related psychosis 4.7\% (2.4-7.0), unipolar depression $5.6 \% \quad(2.2-9.0)$, bipolar disorder $4.9 \%$ (0.7-9.1), and cluster B personality disorder $6.6 \%$ (3.0-10.0). The difference between diagnostic groups was not statistically significant.

Table 2 reports the pattern and age at the onset of gambling in the sample studied. Out of the 478 patients who gambled in the last year, $77.6 \%$ started to gamble after turning 18 and instant lottery was the most frequent (53.6\%) and first game $(46.0 \%)$ played. Patients mostly gambled in smoke shops $(68.6 \%)$, with a rate of "sometimes during the year" $(56.5 \%)$ or "sometimes during a month" $(26.2 \%)$. The comparison of problem and recreational gamblers showed significant differences in the use of slot machines as the main game $\left(58.4 \%\right.$ vs. $\left.10.3 \% ; \chi^{2}=81.8, p<.001\right)$ and as the first game $\left(31.2 \%\right.$ vs. $\left.10.3 \% ; \chi^{2}=25.1, p<.001\right)$. The temporal pattern of comorbidity in those who were problem gamblers showed that $52.1 \%$ of cases started to gamble before the beginning of their illness.

The sociodemographic and clinical variables associated with CPGI total score in the sample are reported in Table 3. These were male sex, younger age, a family history of gambling, two or more psychiatric diagnoses, alcohol abuse/ dependence, substance abuse/dependence, and tobacco smoking.

All variables with a significant association $(p<.05)$ were entered as independent variables in a linear regression
Table 1. Sociodemographic and clinical features of the sample $(N=900)$

\begin{tabular}{lc}
\hline Variables & $\%(n)$ \\
\hline Sex & \\
Males & $48.3(435)$ \\
Females & $51.7(465)$ \\
Mean age $(S D)$ & $48.7(13.7)$ \\
Race & \\
Caucasians & $96.4(868)$ \\
Others & $3.6(32)$ \\
Education & \\
5th grade & $11.0(99)$ \\
8th grade & $39.2(353)$ \\
11th grade & $18.4(166)$ \\
13th grade & $22.8(205)$ \\
University degree & $8.6(77)$ \\
Marital status & \\
Single & $44.0(396)$ \\
Married & $35.0(315)$ \\
Separated/divorced & $17.0(153)$ \\
Widow/er & $4.0(36)$ \\
Working status & \\
Student & $3.2(29)$ \\
Full-time working & $23.0(207)$ \\
Part-time working & $15.0(135)$ \\
Unemployed & $22.7(204)$ \\
Housewife & $7.1(64)$ \\
Disability pension & $13.4(121)$ \\
Retired & $15.6(140)$ \\
Diagnoses & \\
Schizophrenia and related psychosis & $38.3(345)$ \\
Unipolar depression & $19.8(178)$ \\
Bipolar disorder & $11.4(103)$ \\
Cluster B personality & $20.3(183)$ \\
Anxiety disorders & $6.6(59)$ \\
Others & $3.6(32)$ \\
Family history of gambling & $7.3(66)$ \\
\hline
\end{tabular}

Note. SD: standard deviation.

analysis, where the CPGI total score was the dependent variable (Table 4). The variables that independently and significantly predicted CPGI total score were a family history of gambling disorder, two or more psychiatric diagnoses, drug abuse/dependence, and tobacco smoking.

\section{DISCUSSION}

To our knowledge, this is the first study to investigate the prevalence of at-risk gambling in a large sample of general psychiatry patients, with estimates on the main diagnostic groups.

A comparison of our results to those of other published studies is not easy, since most existing data on the comorbidity of gambling and psychiatric disorders stem from surveys on treatment-seeking gamblers or from community surveys, where common mental disorders like depression and anxiety are the rule. These are definitely different from patients suffering from a major psychiatric disorder attending $\mathrm{CMH}$ centers like those included in this study. 
Table 2. Pattern of gambling in patients cared for by community mental health centers $(N=478)$

\begin{tabular}{lc}
\hline Variable & $\%(n)$ \\
\hline Age at onset of gambling & \\
$\leq 18$ years & $22.4(107)$ \\
$>18$ years & $77.6(371)$ \\
Onset & \\
Gambling onset before psychiatric onset & $55.9(267)$ \\
Gambling onset after psychiatric onset & $44.1(211)$ \\
First type of gambling & \\
Scratch cards & $46.0(220)$ \\
Lottery/lotto & $22.4(107)$ \\
Slot machines & $8.2(39)$ \\
Sports betting & $10.7(51)$ \\
Other & $12.7(61)$ \\
Favorite type of gambling & \\
Scratch cards & $53.6(256)$ \\
Lottery/lotto & $19.7(94)$ \\
Slot machines & $12.8(61)$ \\
Sports betting & $5.5(27)$ \\
Other & $8.4(40)$ \\
Place of gambling & \\
Bar & $21.4(102)$ \\
Smoke shop & $68.6(328)$ \\
Amusement arcade & $3.6(17)$ \\
Other & $6.4(31)$ \\
Last year gambling frequency & \\
Every day or almost every day & $3.3(16)$ \\
Every week but not every day & $14.0(67)$ \\
Few times a month & $26.2(125)$ \\
A few times a year & $56.5(270)$ \\
\hline
\end{tabular}

Note. ${ }^{\mathrm{a} B o t h}$ recreational and problem gambling.

In a paper that investigated a large sample of psychiatric outpatients with mixed diagnoses, Zimmerman et al. (2006) found a $2.3 \%$ lifetime and $0.7 \%$ current rate of DSM-IV pathological gambling. In a smaller sample of 100 psychiatric inpatients with mixed diagnoses (45 mood disorders and 35 psychotic disorders), Aragay et al. (2012) found a $9.0 \%$ prevalence of gambling difficulties.

As far as single diagnoses, the rate of at-risk gambling found in our sample of schizophrenia patients $(4.7 \%)$ is lower than that reported by Desai and Potenza (2009) who found a $19 \%$ prevalence rate. A sampling selection possibly explains this difference, since only psychotic patients with an "interest in participation" were selected in the New Haven study. In our sample, all schizophrenia patients were included, also when negative symptoms were substantial.

Among the studies that collected patients with mood disorders, Quilty et al. (2011) evaluated 275 patients with a high rate of comorbidity and found a $9.4 \%$ prevalence rate of problem gambling in major depression and 7.3\% in bipolar disorder. The study design of Kennedy et al. (2010) on 579 mood disorder patients was similar to this study and found rates of $12.5 \%$ and $12.3 \%$ of problem gambling in unipolar depression and bipolar disorder, respectively. However, this study recruited subjects not only in psychiatric outpatient clinics but also through advertising.

The Italian Population Survey on Alcohol and other Drugs (IPSAD; Bastiani et al., 2013) is a cross-sectional
Table 3. Sociodemographic and clinical variables and CPGI score

\begin{tabular}{|c|c|c|c|}
\hline Variable & $\begin{array}{l}\text { CPGI score } \\
\text { mean }(S D)\end{array}$ & Test & $(p)$ \\
\hline \multicolumn{4}{|l|}{ Sex } \\
\hline Males & $1.02(3.94)$ & $t=2.40$ & $(.02)$ \\
\hline Females & $0.48(2.79)$ & & \\
\hline Age & - & $r=-.07$ & $(.05)$ \\
\hline \multicolumn{4}{|l|}{ Education } \\
\hline 8th grade or less & $0.67(3.05)$ & $t=0.63$ & $(.53)$ \\
\hline $8+$ grade & $0.81(3.73)$ & & \\
\hline \multicolumn{4}{|l|}{ Income } \\
\hline No income & $0.93(3.95)$ & $t=1.77$ & $(.08)$ \\
\hline Working/retired & $0.53(2.62)$ & & \\
\hline \multicolumn{4}{|c|}{ Psychiatric disorders in childhood } \\
\hline Diagnosed & $0.81(2.95)$ & $t=0.17$ & $(.86)$ \\
\hline Not detected & $0.74(3.45)$ & & \\
\hline \multicolumn{4}{|c|}{ Family history of gambling } \\
\hline Positive & $2.33(5.78)$ & $t=3.48$ & $(<.001)$ \\
\hline Negative & $0.62(3.11)$ & & \\
\hline \multicolumn{4}{|c|}{$2+$ psychiatric diagnoses } \\
\hline $\begin{array}{l}\text { Two or more } \\
\text { diagnoses }\end{array}$ & $1.47(4.71)$ & $t=4.78$ & $(<.001)$ \\
\hline No comorbidity & $0.35(2.33)$ & & \\
\hline \multicolumn{4}{|c|}{ Alcohol abuse/dependence } \\
\hline Abuse/dependence & $1.86(4.80)$ & $t=2.40$ & $(.02)$ \\
\hline $\begin{array}{l}\text { No abuse/ } \\
\text { dependence }\end{array}$ & $0.68(3.30)$ & & \\
\hline \multicolumn{4}{|c|}{ Substance abuse/dependence } \\
\hline Abuse/dependence & $2.27(5.68)$ & $t=3.83$ & $(<.001)$ \\
\hline $\begin{array}{c}\text { No abuse/ } \\
\text { dependence }\end{array}$ & $0.62(3.13)$ & & \\
\hline \multicolumn{4}{|l|}{ Tobacco smoking } \\
\hline Smoking & $1.17(4.31)$ & $t=3.58$ & $(<.001)$ \\
\hline No smoking & $0.36(2.24)$ & & \\
\hline
\end{tabular}

Note. CPGI: Canadian Problem Gambling Index; $S D$ : standard deviation.

Table 4. A linear regression analysis of CPGI total score and associated variables

\begin{tabular}{lcccc}
\hline Variable & Coefficient & \multicolumn{1}{c}{$95 \%$ CI } & $t$ & $p$ \\
\hline $\begin{array}{l}\text { Family history of } \\
\text { problem gambling }\end{array}$ & 1.42 & $0.58-2.27$ & 3.29 & .001 \\
$\begin{array}{l}\text { Two or more } \\
\text { psychiatric }\end{array}$ & 0.66 & $0.15-1.17$ & 2.55 & .011 \\
$\quad$ diagnoses & & & & \\
Tobacco smoking & 0.52 & $0.06-0.98$ & 2.23 & .026 \\
$\begin{array}{l}\text { Substance abuse/ } \\
\text { dependence }\end{array}$ & 0.91 & $-0.02-1.81$ & 1.96 & .05 \\
\hline
\end{tabular}

Note. CPGI: Canadian Problem Gambling Index; CI: confidence interval.

survey that included 31,984 subjects on a representative sample of the Italian population with a proportionalstratified randomized sample. A 5.6\% rate of at-risk gambling was found in previous year gamblers, while in our sample, the rate was almost double (10.1\%). Although a direct comparison with the IPSAD study would need a 
matching of subjects, the difference seems attributable to the presence of high-risk gamblers (CPGI 8+) who were $6.3 \%$ in our sample and $1.6 \%$ in the community survey. Notably, the national sample included adolescents in the 15-17 age range, who are well known to be at a higher risk of gambling.

The lack of significant differences between our diagnostic subgroups was partly unexpected. We hypothesize that major psychiatric disorders such as schizophrenia, bipolar illness, recurrent unipolar depression, and cluster B personality disorder, with an onset in early adulthood and a severe associated disability can produce a cross-diagnostic vulnerability that acts as a risk factor for gambling.

As far as the temporal pattern of comorbidity, half of our patients with at-risk gambling started to gamble before the beginning of the illness. Previous studies have produced mixed results and interpretations on the causal direction of gambling and psychiatric disorders. In the National Comorbidity Survey Replication (Kessler et al., 2008), pathological gambling followed the onset of psychiatric disorders in three fourth of the cases. In the National Epidemiologic Survey on Alcohol and Related Conditions study on a large community sample, designed with a three-year follow-up, it was found that a baseline diagnosis of problematic gambling predicted new-onset mood episodes, generalized anxiety, and alcoholuse disorder (Chou \& Afifi, 2011). A complex pattern of interaction between gambling and depression was found in an 11-year follow-up study of adolescents (Dussault et al., 2016).

Our finding on the association with slot machines gaming is in line with the findings that slot machine gambling is the most troubling type of game among help-seeking problem gamblers (Castrén et al., 2013). Notably, in the IPSAD survey (Bastiani et al., 2013), slot machines showed the highest association with gambling severity among both onegame players and multigame players (odds ratios equal to 4.3 and 4.5 , respectively).

The associations of problem gambling with substance abuse/dependence and tobacco smoking replicate the findings on mental health comorbidity of previous surveys. A meta-analysis on the issue (Lorains, Cowlishaw, \& Thomas, 2011) found a high prevalence of comorbid disorders, such as nicotine dependence (60.1\%) and substance-use disorder $(57.5 \%)$, with a wide heterogeneity in estimates across studies for substance-use disorders $(26.0 \%-76.3 \%)$.

Some limits of this study must be pointed out. The selection of the CPGI 3/4 cut-off to identify at-risk gambling was criticized (Currie, Hodgins, \& Casey, 2013) for collapsing the "moderate" at risk category (score: 3-8) and the "true" problem gambling class (scores of $8+$ ), because the "moderate risk" was not robust in the association with clinical variables. However, Canale et al. (2016) reported in a large community survey in Great Britain that most "low-risk gamblers" (CPGI 1-4) exhibited dependence harms and social harms similar to problem gamblers and found it necessary to consider harms experienced at any level of gambling involvement. In this study, prevalence rates are reported according to different cut-offs, including the 4/5 one.

Our rates could be compared only between diagnostic groups, but we had not a true control group, with the main indirect reference being a large Italian population survey.
However, none of the published studies, both on single diagnoses or in mixed groups, had controls without psychiatric disorders, and, when available, the comparison was made with community surveys in the same countries (Haydock, Cowlishaw, Harvey, \& Castle, 2015; Jones et al., 2015; Kennedy et al., 2010; Zimmermann et al., 2006).

Another limitation could be the possible co-occurrence of a major psychiatric diagnosis with another, like borderline personality disorder and depression, so that the boundaries between our main diagnostic classes could be more blurred than what was supposed. Anyway, clinicians had to make a choice about what was the primary diagnosis, and some overlapping, like schizophrenia and borderline personality disorder are unlikely. Patients with a diagnosis of schizoaffective disorder were not included to avoid this possible bias.

Finally, data on the cognitive functioning of our patients were not available, although dysfunctions in some cognitive domains, like working memory and inhibition, have been associated with gambling behavior (Potenza et al., 2003; Roca et al., 2008).

\section{CONCLUSIONS}

We found a substantial rate of at-risk gambling in a large clinical psychiatry sample, which may be lower than that found in less severe psychiatric morbidity, but definitely higher than that found in the general population. Our data suggest the opportunity to devise a screening routine for gambling in patients attending general psychiatric facilities, in order to detect and treat an unmet need and possibly prevent the personal and social consequences of this comorbidity.

Among future directions, we are planning a follow-up of our patients with at-risk gambling to evaluate the temporal stability of the diagnosis, to identify risk factors for a chronic course of the comorbidity, and its effects on global outcome measures. We are also planning the comparison of our prevalence estimates with those of subjects taken from the large IPSAD community survey, matching for the main sociodemographic variables in order to accurately estimate the risk of belonging to a population with severe mental illness.

Funding sources: No financial support was received for this study.

Authors' contribution: AB: study concept and design, analysis, and interpretation of data. CT: analysis and interpretation of data, statistical analysis, and study supervision. FB: statistical analysis. AT: monitoring of data and interpretation. PV: analysis and interpretation of data. ES: study concept and design and study supervision. AV: analysis and interpretation of data and study supervision. All authors had full access to data in the study and take responsibility for the integrity of the data and the accuracy of data analysis. 
Conflict of interest: The authors declare no conflict of interest.

Acknowledgements: The authors would like to thank Dr. Elisabetta Carpi and Dr. Alessandro Martinelli for their precious help in collecting data.

\section{REFERENCES}

American Psychiatric Association. (2000). Diagnostic and statistical manual of mental disorder (DSM-IV-TR) (text rev.). Washington, DC: American Psychiatric Association.

Aragay, N., Roca, A., Garcia, B., Marqueta, C., Guijarro, S., Delgado, L., Garolera, M., Alberni, J., \& Vallès, V. (2012). Pathological gambling in a psychiatric sample. Comprehensive Psychiatry, 53(1), 9-14. doi:10.1016/j.comppsych.2011.02.004

Bastiani, L., Gori, M., Colasante, E., Siciliano, V., Capitanucci, D., Jarre, P., \& Molinaro, S. (2013). Complex factors and behaviors in the gambling population of Italy. Journal of Gambling Studies, 29(1), 1-13. doi:10.1007/s10899-011-9283-8

Bonnaire, C., Kovess-Masfety, V., Guignard, R., Richard, J.B., du Roscoät, E., \& Beck, F. (2017). Gambling type, substance abuse, health and psychosocial correlates of male and female problem gamblers in a nationally representative French sample. Journal of Gambling Studies, 33, 343-369. doi:10.1007/ s10899-016-9628-4

Canale, N. L., Vieno, A. L., \& Griffiths, M. D. (2016). The extent and distribution of gambling-related harms and the prevention paradox in a British population survey. Journal of Behavioral Addictions, 5(2), 204-212. doi:10.1556/2006.5.2016.023

Castrén, S., Basnet, S., Pankakoski, M., Ronkainen, J. E., Helakorpi, S., Uutela, A., Alho, H., \& Lahti, T. (2013). An analysis of problem gambling among the Finnish working-age population: A population survey. BMC Public Health, 13(1), 519. doi:10.1186/1471-2458-13-519

Chou, K. L., \& Afifi, T. O. (2011). Disordered (pathologic or problem) gambling and axis I psychiatric disorders: Results from the National Epidemiologic Survey on Alcohol and Related Conditions. American Journal of Epidemiology, 173(11), 1289-1297. doi:10.1093/aje/kwr017

Colasante, E., Gori, M., Bastiani, L., Siciliano, V., Giordani, P., Grassi, M., \& Molinaro, S. (2013). An assessment of the psychometric properties of Italian version of CPGI. Journal of Gambling Studies, 29, 765-774. doi:10.1007/s10899-012-9331-z

Cowlishaw, S., Gale, L., Gregory, A., McCambridge, J., \& Kessler, D. (2017). Gambling problems among patients in primary care: A cross-sectional study of general practices. British Journal of General Practice, 67(657), e274-e279. doi:10.3399/ bjgp17X689905

Currie, S. R., Hodgins, D. C., \& Casey, D. M. (2013). Validity of the Problem Gambling Severity Index interpretive categories. Journal of Gambling Studies, 29(2), 311-327. doi:10.1007/ s10899-012-9300-6

Desai, R. A., \& Potenza, M. N. (2009). A cross-sectional study of problem and pathological gambling in patients with schizophrenia/schizoaffective disorder. Journal of Clinical Psychiatry, 70(9), 1250-1257. doi:10.4088/JCP.m04359

Dussault, F., Brendgen, M., Vitaro, F., Carbonneau, R., Boivin, M., \& Tremblay, R. E. (2016). Co-morbidity between gambling problems and depressive symptoms: A longitudinal perspective of risk and protective factors. Journal of Gambling Studies, 32(2), 547-565. doi:10.1007/s10899-015-9546-x

Ferris, J., \& Wynne, H. (2001). The Canadian Problem Gambling Index: Final report. Ottawa, ON: Canadian Centre on Substance Abuse.

Haydock, M., Cowlishaw, S., Harvey, C., \& Castle, D. (2015). Prevalence and correlates of problem gambling in people with psychotic disorders. Comprehensive Psychiatry, 58, 122-129. doi:10.1016/j.comppsych.2015.01.003

Jones, L., Metcalf, A., Gordon-Smith, K., Forty, L., Perry, A., Lloyd, J., Geddes, J. R., Goodwin, G. M., Jones, I., Craddock, N., \& Rogers, R. D. (2015). Gambling problems in bipolar disorder in the UK: Prevalence and distribution. Brish Journal of Psychiatry, 207(4), 328-333. doi:10.1192/bjp.bp.114.154286

Kennedy, S. H., Welsh, B. R., Fulton, K., Soczynska, J. K., McIntyre, R. S., O’Donovan, C., Milev, R., le Melledo, J. M., Bisserbe, J. C., Zimmerman, M., \& Martin, N. (2010). Frequency and correlates of gambling problems in outpatients with major depressive disorder and bipolar disorder. Canadian Journal of Psychiatry, 55(9), 568-576. doi:10.1177/ 070674371005500905

Kessler, R. C., Hwang, I., LaBrie, R., Petukhova, M., Sampson, N. A., Winters, K. C., \& Shaffer, H. J. (2008). DSM-IV pathological gambling in the National Comorbidity Survey Replication. Psychological Medicine, 38, 1351-1360. doi:10.1017/ S0033291708002900

Lorains, F. K., Cowlishaw, S., \& Thomas, S. A. (2011). Prevalence of comorbid disorders in problem and pathological gambling: Systematic review and meta-analysis of population surveys. Addiction, 106(3), 490-498. doi:10.1111/j.13600443.2010.03300.x

McIntyre, R. S., McElroy, S. L., Konarski, J. Z., Soczynska, J. K., Wilkins, K., \& Kennedy, S. H. (2007). Problem gambling in bipolar disorder: Results from the Canadian Community Health Survey. Journal of Affective Disorders, 102(1-3), 27-34. doi:10.1016/j.jad.2006.12.005

Potenza, M. N., Leung, H. C., Blumberg, H. P., Peterson, B. S., Fulbright, R. K., Lacadie, C. M., Skudlarski, P., \& Gore, J. C. (2003). An FMRI Stroop task study of ventromedial prefrontal cortical function in pathological gamblers. American Journal of Psychiatry, 160(11), 1990-1994. doi:10.1176/appi.ajp. 160.11 .1990

Quilty, L. C., Watson, C., Robinson, J. J., Toneatto, T., \& Bagby, R. M. (2011). The prevalence and course of pathological gambling in the mood disorders. Journal of Gambling Studies, 27(2), 191-201. doi:10.1007/s10899-010-9199-8.

Roca, M., Torralva, T., López, P., Cetkovich, M., Clark, L., \& Manes, F. (2008). Executive functions in pathologic gamblers selected in an ecologic setting. Cognitive Behavioral Neurology, 21(1), 1-4. doi:10.1097/WNN.0b013e3181684358

Rodriguez-Monguio, R., Errea, M., \& Volberg, R. (2017). Comorbid pathological gambling, mental health, and substance use disorders: Health-care services provision by clinician specialty. Journal of Behavioral Addictions, 6(3), 406-415. doi:10.1556/ 2006.6.2017.054

Rossi, A., Alberio, R., Porta, A., Sandri, M., Tansella, M., \& Amaddeo, F. (2004). The reliability of the Mini-International Neuropsychiatric Interview - Italian version. Journal of Clinical Psychopharmacology, 24(5), 561-563. doi:10.1097/01. jcp.0000139758.03834.ad 
Sheehan, D. V., Lecrubier, Y., Sheehan, K. H., Amorim, P., Janavs, J., Weiller, E., Hergueta, T., Baker, R., \& Dunbar, G. C. (1998). The Mini-International Neuropsychiatric Interview (M.I.N.I.): The development and validation of a structured diagnostic psychiatric interview for DSM-IV and ICD-10. Journal of Clinical Psychiatry, 59(Suppl. 20), 22-33.

Wareham, J. D., \& Potenza, M. N. (2010). Pathological gambling and substance use disorders. American Journal of Drug and Alcohol Abuse, 36(5), 242-247. doi:10.3109/00952991003721118
Williams, R. J., \& Volberg, R. A. (2014). The classification accuracy of four problem gambling assessment instruments in population research. International Gambling Studies, 14(1), 15-28. doi:10.1080/14459795.2013.839731

Zimmerman, M., Chelminski, I., \& Young, D. (2006). Prevalence and diagnostic correlates of DSM-IV pathological gambling in psychiatric outpatients. Journal of Gambling Studies, 22(2), 255-262. doi:10.1007/s10899-006-9014-8 